\title{
Les « métis franco-indigènes » dans le second Empire colonial
}

\author{
Yerri Urban
}

Dernière version avant publication de la contribution publiée in Sylvie Laurent \& Thierry Leclère (dir.), De quelle couleur sont les Blancs?, Paris, La Découverte, 2013, p.121-127.

En droit français, le «Blanc » a cessé d'être une catégorie juridique en 1831 pour les droits civils, en 1833 pour les droits politiques. En effet, le «préjugé de couleur », ce système de discriminations raciales entre Blancs, forcément libres, et gens de couleur, libres ou esclaves, qui existait depuis le XVIII ${ }^{\mathrm{e}}$ siècle dans les colonies françaises, a pris fin (il avait été supprimé une première fois entre 1792 et 1802). Comparable parfois à l'apartheid (interdiction des mariages mixtes, de la reconnaissance d'enfants naturels, etc.), le préjugé de couleur a semblé condamné à l'oubli avec la seconde abolition de l'esclavage de 1848, abolition générale cette fois, qui a mis fin à la coïncidence entre couleur de peau et condition servile [Niort, 2007 ; Régent, 2007]. L'autre législation raciale de l'histoire du droit français, celle de Vichy, fut quant à elle avant tout une machine à racialiser des populations perçues comme blanches : les juifs, qu'ils soient français, étrangers ou indigènes [Gros, 1996], mais aussi les indigènes musulmans d'Algérie.

Pendant le second Empire colonial, si la race était très présente dans les têtes, elle l'était peu dans le droit: la frontière entre Français et indigènes, de toute évidence influencée par un droit romain qui n'avait pas besoin d'idéologie raciste, reposait sur le droit de la nationalité [Urban, 2010]. Le rôle de la race était secondaire et servait principalement à faire la distinction entre les différents peuples indigènes. Dans ce contexte, l'apparition d'une «question des métis », issus de Blancs et d'indigènes « de couleur », est apparue pour le moins incongrue : il existait une véritable fonction publique française « de couleur » dans les 
colonies, et il n'y avait pas de problème juridique dans le cadre d'un droit aveugle à la race sur ce point. Ce paradoxe ne cessera d'être constaté par tout ou partie des juristes et trouvera un aboutissement singulier avec, dans le même temps, la consécration partielle du «Blanc» comme catégorie juridique et le refus partiel de le consacrer. Comment en est-on arrivé là ? De la situation a-raciale des enfants issus d'unions mixtes, on est passé au triomphe de l'ambiguïté pour les métis d'Indochine [Saada, 2007], jusqu'à cette consécration (pour les étrangers) et ce refus de consécration (pour les Français) de la blancheur, en Afrique noire et à Madagascar.

\section{Les enfants issus d'unions mixtes}

Dans le second Empire colonial, la principale distinction entre personnes passait par la nationalité : d'un côté les membres du peuple de la métropole, les Français (qu'on appelait aussi « citoyens français », en référence au modèle de la citoyenneté romaine), de l'autre les originaires des territoires colonisés, les indigènes, qui pouvaient être, selon leur territoire d'origine, sujets français (colonies), protégés français (protectorats) ou administrés français (pays sous mandats B de la Société des Nations). Le droit colonial de la nationalité comportait ainsi trois catégories : le Français, l'étranger et l'indigène. D’un côté, il y avait un droit de la nationalité qui déterminait notamment l'acquisition de la nationalité française à la naissance et les modalités d'acquisition de la nationalité française par les étrangers, les conséquences des mariages entre Français et étrangers et la situation des enfants issus des unions mixtes; de l'autre côté, chaque peuple indigène était soumis à son droit de la nationalité propre et ses membres ne pouvaient accéder que très difficilement à la nationalité française. Généralement, l'indigène était défini négativement comme un non-Français nonétranger, mais dans le cas de l'Indochine, une législation élaborée est apparue dès les années 1880. Je ne me préoccuperai pas ici de l'Afrique du Nord, dont la population indigène était considérée comme blanche, ou en tout cas comme n'étant pas une population « de couleur», mais des territoires où les indigènes relevaient du ministère des Colonies, c'est-à-dire les 
colonies d'Afrique noire, de Madagascar, d'Asie et d'Océanie (la Guyane étant un cas à part). Les principes juridiques adoptés pour les enfants issus d'unions mixtes n'avaient rien de raciaux. Il s'agissait d'articuler plusieurs hiérarchies : la hiérarchie des sociétés, avec la supériorité des peuples regardés par le droit international public et par le droit colonial comme civilisés, c'est-à-dire soumis à un droit de type occidental (européens, américains et japonais ) sur les peuples regardés comme non civilisés, la supériorité de l'homme sur la femme (et avant tout du mari sur l'épouse), la supériorité du Français sur l'étranger et sur l'indigène.

Il n'y a jamais eu de législation relative aux mariages mixtes ailleurs que dans les protectorats d'Indochine (Annam, Tonkin, Cambodge). C'était donc à la jurisprudence de combler les vides. Les principes en furent élaborés en Algérie : la femme indigène acquérait la nationalité française de son mari (phénomène non négligeable en Indochine), la femme française qui épousait un indigène conservait sa nationalité. Ces principes se retrouveront dans les protectorats d'Afrique du Nord et dans la jurisprudence et la législation indochinoises. La hiérarchie des sociétés l'emportait sur le principe de la supériorité de l'homme sur la femme. Avec deux exceptions, toutefois : au Cambodge de 1920 à 1934, la femme française suivait la condition de son mari cambodgien - l'enfant ne pouvait donc être que cambodgien; en Annam à partir de 1936, la femme française pouvait opter pour la nationalité de son mari annamite : si elle le faisait, l'enfant ne pouvait être qu'annamite.

L'enfant légitime suivait partout la condition de son père français. Les choses étaient plus complexes quand la mère était française : si le père était indigène sujet français, l'enfant était français, comme sa mère ; en revanche, si le père était indigène protégé français, l'enfant était indigène protégé français comme son père. Toutefois, à partir de 1930, ce principe a changé en Indochine: l'enfant de père indigène protégé était français comme sa mère française, mais cela pouvait provoquer des situations de double nationalité franco-indigène au Cambodge et au Tonkin jusqu'au milieu des années 1930.

Il en allait autrement des enfants naturels reconnus, dont la situation était complexe : l'enfant suivait la nationalité du parent à l'égard duquel la filiation avait été établie en premier, donc la mère indigène dans la grande majorité des cas. La législation fut modifiée en 3 
1916 et 1918 pour permettre aux pères français de transmettre systématiquement leur nationalité à l'enfant qu'ils avaient reconnu. En contrepartie, elle autorisait le ministère public à poursuivre, d'office, l'annulation de toute reconnaissance par un Européen d'un enfant naturel indigène. Par la suite, à partir de 1928-1930, le parent français transmettait systématiquement sa nationalité à son enfant, mais cela pouvait, là encore, provoquer des situations de double nationalité franco-indigène au Cambodge et au Tonkin jusqu'au milieu des années 1930. On restait ici dans le cadre d'un droit de la nationalité hiérarchisé, mais où la notion de race, et a fortiori la notion de blancheur, n'entrait pas en ligne de compte. Il en alla autrement à partir du moment où la question des enfants naturels qui n'avaient pas été reconnus fut abordée.

\section{Les métis d'Indochine : le triomphe de l'ambiguïté}

Le passage à une approche raciale en Indochine doit être replacé dans son contexte : il y existait une distinction symétrique entre populations du point de vue du seul droit de la nationalité ; au Français et à l'étranger s'opposaient l'indigène et l'étranger assimilé à l'indigène. Or, dès la fin $\mathrm{du} \mathrm{XIX}^{\mathrm{e}}$ siècle, est apparue et s'est développée une conception de l'étranger assimilé et de l'indigène sanctionnant une lecture de la mosaïque ethnique en terme nationaux et raciaux, où la race était conçue comme mi-ethnique, mi-biologique. En effet, les autorités coloniales considéraient bien souvent que seuls étaient indigènes les membres de la race-ethnie dominante du territoire, qu'ils soient Viêt ou Khmer, ce qui aurait pour conséquence de transformer en apatrides les membres des minorités ethniques qui ne pouvaient pas se placer sous la protection d'un État asiatique étranger (la Chine et le Siam en l'espèce). Mais la Cour de cassation rejeta cette conception en 1905. Les nationalités indochinoises furent ainsi conçues comme l'amalgame d'une race-ethnie dominante et de minorités. Toutefois, à partir de la fin des années 1920, le phénomène multi-ethnique fut de moins en moins perçu en termes biologiques et l'évolution du droit international public entraîna un traitement égalitaire pour la Chine et le Siam. 
Revenons aux métis. C'est à partir du moment où le père français ne reconnaissait pas l'enfant que l'on passait à une logique raciale. À partir de 1897, la législation prévoyait qu'était français l'individu né de parents inconnus, mais elle n'était pas applicable aux indigènes. Il aurait été possible de s'en tenir au seul critère de la société dans laquelle l'enfant de parents inconnus avait été socialisé pour déterminer son statut juridique, sans tenir compte de son apparence physique. Toutefois, le juge colonial préférait retenir le critère de la race, mais ses conséquences sur le statut évoluèrent : dans les années 1900, l'enfant d'apparence eurasienne devait être indigène, à partir des années 20, il devait être français. Plusieurs conceptions se sont affrontées : deux d'entre elles consistaient à s'en tenir au critère «biologique », c'est à dire au critère du seul aspect physique, complété par une expertise médico-légale en cas de doute. À l'opposé, une autre conception consistait à refuser tout recours à un critère racial, et n'acceptait que le recours au critère de la socialisation.

Ce débat montre bien la grande différence de perspective avec le préjugé de couleur ou avec la législation antisémite qui viendra par la suite : dans ces deux cas, la race est une pure construction juridique, une pure fiction juridique, qui ne sanctionne pas la croyance en l'existence d'une race biologique. Dans le cas du préjugé de couleur, la véritable question est celle du statut des descendants d'esclaves et des affranchis, et comme on le sait, des personnes juridiquement blanches pouvaient ne pas en avoir l'apparence physique; dans le cas de la législation antisémite, la race est construite à partir de la religion des grands-parents. Ici, le droit est censé prendre en compte la race biologique.

Une position «médiane ", qui l'a emporté, consistait à retenir les deux critères, sociologiques et biologiques. Mais ces deux critères devaient-ils se cumuler où étaient-ils interchangeables ? C'est la deuxième solution qui fut retenue : en 1928, fut adopté un décret permettant aux individus nés en Indochine, dont l'un des parents était inconnu, de se voir reconnaître par un tribunal la qualité de Français s'il était présumé de « race française ». Les travaux préparatoires montrent que l'on a affaire ici à un chef-d'œuvre d'ambiguïté : il est tout autant possible de faire une lecture purement sociologique de l'expression qu'une lecture purement biologique. C'est la deuxième solution qui l'a emporté dans la pratique, alors même que le mot «race» fut exclu des textes postérieurs et analogues. La blancheur est ici 
implicite, elle est le produit d'un consensus interprétatif.

Mais le législateur indochinois s'est alors préoccupé des «métis» de Chinois et d'indigènes, et est passé de la question de la race biologique à celle de l'ethnicité. De fait, le parcours fut inverse de celui effectué pour les métis franco-indigènes : l'approche était de moins en moins raciale. Après avoir regardé ces enfants de Chinois et d'indigènes comme des Asiatiques étrangers tout en leur reconnaissant un statut proche de celui des indigènes, le colonisateur, invoquant le danger que représenterait leur allégeance effective à la Chine et leur transformation en étrangers de droit commun, obtint en 1933 l'adoption d'un décret (qui sera repris en Océanie en 1933 et en 1937 en Nouvelle-Calédonie) qui modifiait substantiellement les conceptions antérieures de l'indigène : dans un contexte où la nationalité française se transmettait aussi bien par le père que par la mère, était indigène tout enfant légitime ou naturel né en Indochine dont l'un des parents était indigène, à moins que l'autre parent n'ait été français ou présumé français. Tout le paradoxe du texte tient donc dans la manière dont il sanctionne la capacité d'absorption des sociétés indigènes tout en tendant à considérer de plus en plus les Français comme les membres d'une ethnie européenne de race blanche. La conception ethnique-raciale qui prévalait auparavant pour les nationalités indochinoises fut abandonnée pour ces dernières, mais tendait à être reprise pour la nationalité française. Cette transposition avait toutefois ses limites, la législation indochinoise étant la synthèse de courants opposés : comme on l'a vu, en Annam, à partir de 1936, la femme française qui optait pour la nationalité de son mari annamite transmettait la nationalité annamite à son enfant considéré comme eurasien...

\section{Les métis d'Afrique noire et de Madagascar : une consécration du Blanc?}

Dans le cas des métis d'Indochine, les débats lors de l'élaboration du texte, mais aussi l'environnement régional, avec l'assimilation des Japonais (dans les années 1890) puis des Chinois et des Siamois (dans les années 1930) aux «étrangers de droit commun » (et non aux étrangers assimilés aux indigènes), avait empêché la formulation explicite du recours au 
critère de la blancheur, ou plus exactement, de l'ascendance blanche. Il en allait autrement des territoires qui n'étaient entourés que de colonies européennes. C'est à l'occasion de l'élaboration du décret de 1930 sur les métis en Afrique occidentale française (AOF) qu'eut lieu le véritable débat sur la formulation de la blancheur. À la différence de ce qui s'était passé en Indochine, les métis devaient ici remplir à la fois les critères biologiques et sociologiques dans le cadre d'une procédure plus complexe, mi-administrative, mi-judiciaire. La formulation du critère biologique a focalisé les débats, comme en témoigne la liste des différentes formules envisagées : l'ascendant inconnu devait être « de race blanche de souche européenne », «d'origine française ou d'origine étrangère de race blanche », «d'origine française ou de souche européenne », «de souche européenne, d'origine française ou étrangère $\gg . .$. Les raisons de ces multiples hésitations sont simples : la référence à la seule race blanche ou à la seule origine européenne écartait «les fils de citoyens français de couleur », «les enfants des Français de La Réunion et des Antilles »; quant à la référence à l'origine européenne, elle visait à exclure les métis de «Syriens» ou de «Nord-Africains ». Finalement, après un imbroglio, on opta pour la formule «d'origine française ou étrangère de souche européenne »; cette formulation sera reprise dans d'autres territoires préférant par contre, quant à la procédure, le système indochinois (Madagascar en 1931, NouvelleCalédonie en 1933, Afrique équatoriale française en 1936, Togo en 1937, Cameroun en 1944).Ainsi, en théorie, lorsque le père qui n'avait pas reconnu l'enfant était français, l'apparence physique, la couleur n'était pas prise en compte, alors que c'était le cas lorsque le père était étranger... Dans la pratique, c'est l'apparence physique qui sera là encore prise en compte.

Le débat sur les métis d'Afrique occidentale française n'en a pas moins montré les limites d'une approche faisant dépendre le statut juridique de l'apparence physique: d'une part, elle ne pouvait aboutir qu'à une remise en cause de la nationalité des «Français de couleur », d'autre part, comme l'avait souligné un administrateur colonial farouchement opposé à ces textes : «Prendre, comme critérium du statut juridique applicable, le seul facteur de race est logiquement impraticable » [Hoeffel, 1932, p. 92]. L'introduction du critère racial pour accéder à la nationalité française n'est pas allée au-delà des métis de parents inconnus... 


\section{Bibliographie}

Gros D. (dir.) (1996), Le droit antisémite de Vichy, Le genre humain, $\mathrm{n}^{\circ} 30-31$, Le Seuil, Paris.

HoEfFel E. (1932), De la condition juridique des étrangers au Cambodge, Ch. Hiller, Strasbourg.

Niort J.-F. (dir.) (2007), Du Code noir au Code civil. Jalons pour l'histoire du droit en Guadeloupe, L'Harmattan, Paris.

REGENT F. (2007), La France et ses esclaves. De la colonisation aux abolitions (16201848), Grasset, Paris.

SAADA E. (2007), Les Enfants de la colonie. Les métis de l'Empire colonial entre sujétion et citoyenneté, La Découverte, Paris.

URBAN Y. (2010), L'Indigène dans le droit colonial français (1865-1955), LGDJ, coll. «Fondation Varenne », Paris. 